\title{
Diffusion Process of Mobile Social Shopping Based on LINE Service
}

\author{
Wei-Shang Fan*, Jan-Kai Huang \\ Department of Business Administration, Nanhua University, Taiwan
}

Copyright $(2016$ by authors, all rights reserved. Authors agree that this article remains permanently open access under the terms of the Creative Commons Attribution License 4.0 International License

\begin{abstract}
It seems that Line has become the most commonly used instant messaging software. Over 44 million people are sending messages through Line everyday with one billion messages flowing between mobile phones and computers in the world. Why Line would be so popular in Taiwan and people are obsessed by it? For what reason the software could attract people to use and set up discussing group to further create unlimited demands? The topic is worthy of exploring and investigating. To better understand the crucial factors of behavior intention in use of activity community service, this study is intended to investigate what kinds of factors effect of using LINE service on consumers' attitude towards in activity community market. The research approach includes the bass SEM model and catastrophe model. We explore the relationships between satisfaction, switching barriers and behavior intention through by CFA and SEM. Secondly, GEMCAT is employed to estimate the cusp catastrophe model using our research data. The qualitative model was instrumental in planning a strategy for a changing market environment characterized by inflation and decreasing switching cost. With high switching cost, whether a person is satisfied or dissatisfied, it is very difficult to change to the opposite state. Finally, we discuss the findings from an academic and managerial perspective and provide directions for future research.
\end{abstract}

Keywords Line, Trust, Attitude, Perceived Usefulness, Catastrophe Model

\section{Introduction}

Line (styled "LINE") is a proprietary application for instant communications on electronic devices. Line users exchange texts, images, video and audio, and conduct free VoIP conversations and video conferences. Recently, the "Line" also provides several different kinds of e-commerce and online shopping service for their member, such as stickers, game and online shopping. Comparing to other transaction modes, it is easier for consumers to aggregate their demands in virtual communities. The coherence and trust among community members, at the same time, further reduce potential risks in transactions.

With the evolution of technology, the smart phone has changed the way people communicate and has driven instant messenger boom, such as "LINE messenger" which has a staggering number of users in the world. In recent years, the emergence of the Internet as a popularly acceptable media platform has changed the way how information transmission and communication were conducted. Virtual community provides a channel to share information. The engagement behaviors of enterprises or individual make people have more willingness to join virtual community. Sharing information from enterprises or individual can also improves the interactive relationship with other users. Line provide the real-time voice and video calls with friends are free with LINE. Currently, it is available on iPhone, Android, Windows Phone, BlackBerry (Voice Call) and PC (Windows / MacOS). Fig. 1 shows the Line service.

Based on the above-mentioned research background, the purpose of this study is to investigate the significant factors used by Line service, it includes: (1) to explore the relationship among the trust, attitude to use and behavior intention of Line; (2) describe the nonlinear relationship between attitude to use and perceived usefulness based on different degree of trust using by the cusp catastrophe model. Finally, we will develop a loyalty strategy for a Line choice behavior in the Line market.

To improve our understanding of Line users' decision-making process, a model that considers Line's perceived usefulness, trust and attitude to use is established. Based on the literatures review (Wang and Qualls, 2007; Hsu and Lon, 2008), the hypothesis and measurement model are formulated for the exogenous variable and the endogenous variables as shown in the following hypothesis, and to explain the relationships among Trust (TR), Attitude (AT) and Perceived Usefulness (PU). The following seven hypotheses are formulated as:

H1: Trust has positive effects on behavior intention.

$\mathrm{H} 2$ : Perceived usefulness has positive effects on behavior intention. 


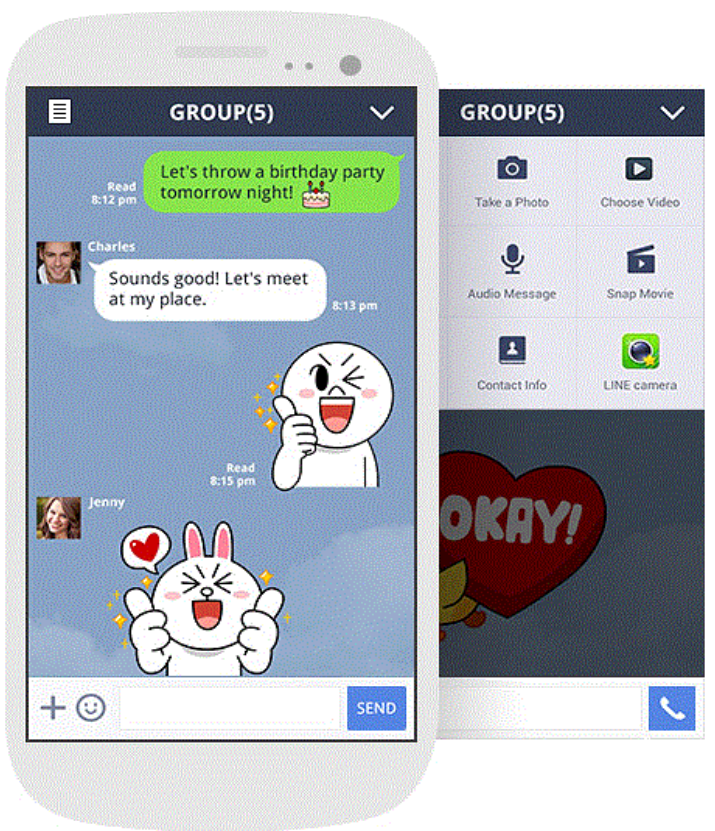

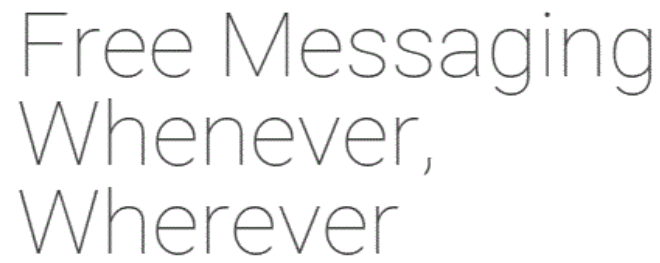

Exchange free instant messages with friends whenever and wherever with one-on-one and group chats. LINE is available on all smartphone devices (iPhone, Android, Windows Phone, Blackberry, Nokia) and even on your PC

Figure 1. The Line Service

\section{Review of Cusp Catastrophe Theory}

The catastrophe structure most commonly has been applied the cusp model (Gresov, Haveman, \& Oliva 1993). Cusp catastrophe model can be formalized by potential or gradient structures, a potential function $\mathrm{F}(\mathrm{x}, \mathrm{c})$ is a function of both the system state $\mathrm{x}$ and the control parameter(s) c. In mathematical terminology, the Cusp Catastrophe Model (CCM) consists of one behavior variable and only two control variables. The three dimensional phase space (see Fig. 1) of the cusp model can be described by the following potential equation:

$$
F(v, u, x)=\frac{1}{4} x^{4}+\frac{1}{2} u x^{2}+v z
$$

Where $x$ and $y$ are the two control dimensions and $z$ represents the behavior surface. The equilibrium surface $M$ is determined by $\mathrm{Eq}(2)$ :

$$
\begin{aligned}
& \frac{\partial F}{\partial x}=z^{3}+y z+x=0 \\
& M_{F}:\left\{(x, y, z) \mid z^{3}+y z+x=0\right\}
\end{aligned}
$$

The values of $\mathrm{x}$ in correspondence to which attains a local maximum or minimum satisfaction of the condition as in:

$$
3 z^{2}+y=0
$$

To obtain the bifurcation set, project the equilibrium surface $\mathrm{M}$ into the control space by eliminating $\mathrm{x}$ from Eq. (2) and Eq. (3):

$$
k=\left\{(x, y): 4 y^{3}=27 x^{2}\right\}
$$

The Hessian discriminate is expressed with the symbol " $K$ ". This allows us to examine how the potential function looks in various regions of parameter space as well as in the set $\mathrm{K}$ itself. When the Hessian discriminate is greater than zero, the system only has a steady equalization point; if Hessian discriminate is smaller than zero, the system will have two steady equalization points and an unstable equalization point. The cusp catastrophe occurs in systems whose behavior is dependent two control factors. Its graph is three-dimensional with every point on the surface representing an equilibrium state. The parameter $y$ determines whether the system has one or can have two stable equilibria. When $y>0$ only one stable equilibrium can exit whatever the value of $\mathrm{v}$. When $\mathrm{y}<0$ it depends upon the value set of $(x, y)$ whether the system has a single low level of stable equilibrium, or a low level and a high level equilibria, or a single high level of equilibrium.

GEMCAT approaches have been successfully applied in a number of different organizational research contexts (e.g. Gresov, 1987; Oliva, 1992). Oliva et al.'s (1987) GEMCAT approach allows all variables in a catastrophe to be latent composites. To accomplish this, the variable $\mathrm{X}, \mathrm{Y}$, and $\mathrm{Z}$ in the canonical cusp is presented by Eq. (5)

$$
f(x, y, z)=\frac{1}{4} z^{4}-\frac{1}{2} y z^{2}-x z
$$

The equation (5) can be redefined as these three "latent" unobservable constructs which can thus accommodate univariate or multivariate measurements for each type of variable. This allows the cusp catastrophe model to be rewritten as shown in Eq. (1) and its derivative set equal to zero can be stated as:

$$
f\left(X_{t}^{*}, Y_{t}^{*}, Z_{t}^{*}\right)=\frac{1}{4} Z_{t}^{*^{4}}-X_{t}^{*} Z_{t}^{*}-\frac{1}{2} Y_{t}^{*} Z_{t}^{* 2}
$$


From equation Eq. (6) the estimating goal is to minimize Eq. (7) and Eq. (8):

$$
\begin{aligned}
& \frac{\partial f\left(X_{t}^{*}, Y_{t}^{*}, Z_{t}^{*}\right)}{\partial Z_{t}^{*}}=Z_{t}^{*^{3}}-X_{t}^{*}-Y_{t}^{*} Z_{t}^{*}=0 \\
& \operatorname{Min}_{\alpha i, \beta j, \gamma k} \Phi=\left\|e_{t}^{2}\right\|=\sum_{t=1}^{T}\left[Z_{t}^{* 3}-X_{t}^{*}-Y_{t}^{*} Z_{t}^{*}\right]^{2}
\end{aligned}
$$

where the et $=$ error. That is, for a given empirical data on various specified dependent, splitting, and normal variables, one wishes to estimate the impact coefficients that define their respective latent variables, which make $\Phi$ as close to zero as possible. Minimizing $\Phi$ is equivalent to find the best fitting cusp catastrophe surface to the empirical data.

\section{Data Analysis and Results}

Data collection and Measure Model

To test the hypotheses, an online field survey was conducted. It used a questionnaire designed to be placed on a web site. The online survey yielded 327 usable responses, including 143 males and 184 females. Eighty percent were under 30 years of age and $80 \%$ had at least a college degree, indicating that the respondents were young and educated. All participants indicated that they had some experiences in writing their own Lines and/or reading and commenting on others'. Table 1 summarizes the respondents' profile.

\begin{tabular}{|c|c|c|}
\hline Measure & Items & Percent \\
\hline \multirow[t]{2}{*}{ Gender } & Male & $44 \%$ \\
\hline & Female & $56 \%$ \\
\hline \multirow[t]{3}{*}{ Age } & Under 25 & $68 \%$ \\
\hline & $26-35$ & $28 \%$ \\
\hline & Over 36 & $4 \%$ \\
\hline \multirow[t]{3}{*}{ Location of Taiwan } & North & $45 \%$ \\
\hline & Middle & $25 \%$ \\
\hline & South & $30 \%$ \\
\hline \multirow[t]{3}{*}{ Experience in Lin } & Under 1 year & $5 \%$ \\
\hline & 1 year & $50 \%$ \\
\hline & Over 1 years & $45 \%$ \\
\hline \multirow[t]{5}{*}{ Time in Lin each day } & Under $0.5 \mathrm{~h}$ & $9 \%$ \\
\hline & $0.5 \mathrm{~h}-1 \mathrm{~h}$ & $11 \%$ \\
\hline & $1 \mathrm{~h}-2 \mathrm{~h}$ & $25 \%$ \\
\hline & $2 \mathrm{~h}-3 \mathrm{~h}$ & $25 \%$ \\
\hline & Over $3 \mathrm{~h}$ & $30 \%$ \\
\hline \multirow[t]{2}{*}{ Marital status } & Unmarried & $81 \%$ \\
\hline & Married & $19 \%$ \\
\hline
\end{tabular}

Table 1. Demographic profile

To ensure the content validity of scales, the scale items selected must represent the concept about which generalizations are made. Hence, validated scale items adapted from prior studies were used to measure the constructs of TAM. Additionally, constructs shared by different investigated TAM were measured using the same scale items. All scale items were measured using 5-point Likert-type scale (from $1=$ "strongly disagree" to $5=$ "strongly agree"). This study first developed the measurement model by conducting confirmatory factor analysis (CFA) to measure convergent and discriminate validity. Then, the structural equation model was then estimated for hypotheses testing.

Table 2 indicated the measurement model was first assessed through CFA. The measurement model was further assessed for construct reliability and validity. Construct validity was evaluated by examining the standardized factor loadings within the constructs, average variance extracted (AVE), and the correlation between constructs. Standardized factor loadings on all latent constructs were satisfactory (0.61-0.93), showing satisfactory item convergence on the intended constructs. The AVE of all latent constructs was higher than the suggested value of 0.50 . The above tests indicated that the discriminate validity was upheld for the measurement model. Overall, the confirmatory factor model adequately reflected a good fit to the data. The results of structural equation modeling obtained for the proposed conceptual model revealed a good model fit $(\mathrm{CFI}=0.96$; IFI $=0.96$; $\mathrm{RMSEA}=0.11 ; \mathrm{SRMR}=$ 0.07; NFI $=0.93$ ). Fig. 1 shows the structural model estimates, where the estimate parameters are standardized path coefficients.

Table 2. Consistency reliability and convergent validity of the measurement model

\begin{tabular}{|c|c|c|c|c|}
\hline \multirow{2}{*}{$\begin{array}{c}\text { Constructs } \\
\text { and } \\
\text { indicators }\end{array}$} & \multicolumn{4}{|c|}{ Construct reliability and validity } \\
\cline { 2 - 5 } & Factor & $\boldsymbol{t}$-value & $\begin{array}{c}\text { Average } \\
\text { variance } \\
\text { extracted }\end{array}$ & $\begin{array}{c}\text { Composite } \\
\text { reliabilities }\end{array}$ \\
\hline $\begin{array}{c}\text { Attitude } \\
\text { Towards Use }\end{array}$ & & & 0.92 & 0.77 \\
\hline AT1 & 0.78 & 20.93 & & \\
\hline AT2 & 0.93 & 22.45 & & \\
\hline AT3 & 0.87 & 25.23 & & \\
\hline Trust & & & 0.85 & 0.70 \\
\hline TR1 & 0.89 & 17.28 & & \\
\hline TR2 & 0.90 & 19.89 & & \\
\hline TR 3 & 0.78 & 13.05 & & \\
\hline Perceived & & & 0.91 & \\
\hline Usefulness & & & & \\
\hline PU1 & 0.89 & 18.25 & & \\
\hline PU2 & 0.91 & 21.64 & & \\
\hline PU3 & 0.85 & 17.78 & & \\
\hline
\end{tabular}

Because of the proposed measurement model was consistent with the data, the hypothesis were tested with AMOS 5.0, using the covariance matrix. Fig. 2 portrays the hypotheses test results and the completely standardized 
parameters in the proposed structural model. We assessed overall goodness of fit using the chi-square test. The chi-square test assesses the adequacy of a hypothesized model in terms of its ability to reflect variance and covariance for the data. Fig. 1 shows the structural model estimates, where the estimate parameters are standardized path coefficients. All of our hypothesized associations were significant at $p<0.01$. Fig. 1 shows the standardized path coefficients for our research model. Results indicate that perceived usefulness $(\beta=0.13, p<0.05)$ was a significant determinant of perceived usefulness. Perceived ease of use $(\beta=0.52, p<0.05)$ was a significant determinant of perceived usefulness.

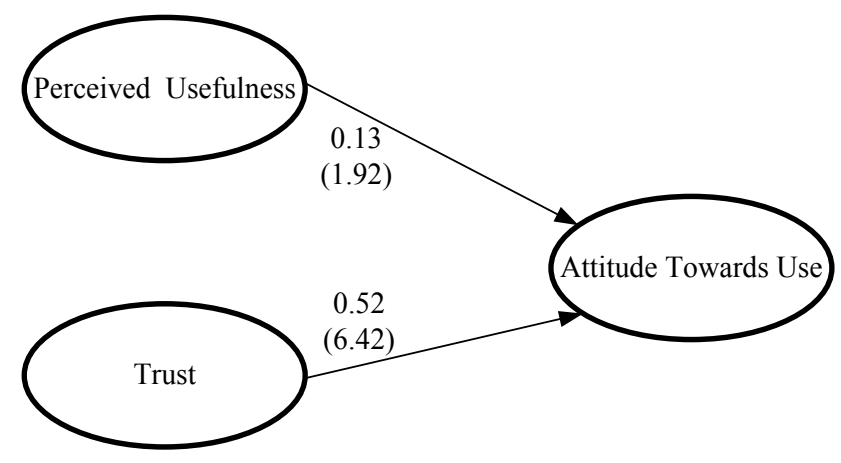

Figure 2. The Result of Structural Equation Model

\section{Model Fit and Discussion}

The control variables ${ }^{1}$ in the model are (1) the normal variable $v$ is defined in terms of "relative perceived usefulness " ( $v_{k}$ with weight $\left.\gamma_{k}\right)$, and (2) and the splitting variable $\mathrm{u}$ uses the "trust" indicator $\left(u_{j}\right.$ with weight $\left.\beta_{j}\right)$, whereas the dependent variable $\mathrm{x}$ is defined in terms of relative attitude towards use $\left(x_{i}\right.$ with weight $\left.\alpha_{i}\right)$. For instance, the latent variables in a cusp catastrophe model take the following general form:

$$
\begin{gathered}
X^{*}=\alpha_{i} \times \Delta x_{i t}, i=1,2,3 ; t=1,2 \ldots 291 \\
u^{*}=\beta_{j} \times \Delta u_{j t}, j=1,2,3 ; t=1,2 \ldots 291 \\
v^{*}=\gamma_{k} \times \Delta v_{k t}, k=1,2 \ldots 3 ; t=1,2 \ldots 291
\end{gathered}
$$

After transforming all variables to $\mathrm{z}$-scores (i.e., $\mathrm{M}=0$, $\mathrm{SD}=1$ ), GENCAT II was employed to fit the hypothesized cusp catastrophe model. Data were fitted using GEMCAT II version 1.3, substitution of these weights into Eq. (9)-Eq. (11). Figure 3 shows the behavior manifold for the cusp catastrophe model. Consider two points at the far edge of the surface (low trust), they represent attitude with the same value of perceived usefulness, but slightly different values of trust. If the value of perceived usefulness increases, the points move forward toward the front of the surface, tracing parallel paths. The figure also shows discontinuous change when a point moves from the left to the right of the surface. At the far edge of the surface, a point can pass smoothly from left to right. But if point is at the front of the surface (high trust), a discontinuous jump will occur at the pleat. At the back of the surface where the trust is small; the attitude is dependent only on its perceived usefulness and increases continuously with it. Increases trust its magnitude lead to a progressively larger divergence between the top and the bottom of the response surface.

If the magnitude of change in u (trust) is small, then a smooth change in $x$ (attitude) would occur, directly proportional to the change in $\mathrm{x}$ (attitude). A small difference in the initial starting positions (e.g., point a and point b) can result in vastly different values for $\mathrm{x}$ (attitude) when the magnitude of $u$ (trust) increases beyond the point where the pleat starts. This phenomenon is illustrated by path A and path $\mathrm{B}$ in Figure 3, where point a is driven downward to point $c$, and point $b$ is driven upward to point $d$. At higher values of $\mathrm{u}$ (trust), however, large changes in $\mathrm{v}$ (perceived usefulness) will produce a sudden discontinuous shift in $\mathrm{x}$ (attitude) as shown in path $\mathrm{C}$, which then contributes to the reverse behavioral result.

It should be noted that once a sudden shift has happened, reversing the values of $\mathrm{v}$ (perceived usefulness) may not cause a substantial downward change in $\mathrm{x}$ (attitude) namely hysteresis. There must be a significant reversal in $\mathrm{v}$ (perceived usefulness) before a shift down to point $f$ would occur. These lags in response are aggravated or mitigated by the size of the u (trust). Within the cusp area, the state variable $\mathrm{x}$ can take on two possible values for a given $(\mathrm{v}, \mathrm{u})$ pair. This characteristic allows for the modeling of lag effects (hysteresis).

\footnotetext{
${ }^{1}$ Our operationalizations of the dependent and independent indicator are measure is as follows

State variable : Attitude : $x^{*}=f\left(x_{1}, x_{2}, x_{3}\right)$

Normal factor: Perceived usefulness : $v^{*}=f\left(v_{1}, v_{2}, v_{3}\right)$

Splitting factor : Trust: $\mathrm{u}^{*}=\mathrm{f}\left(\mathrm{u}_{1}, \mathrm{u}_{2}, \mathrm{u}_{3}\right)$

Ex $: x_{i} \rightarrow$ relative attitude (use of Line service provide by LINE - use of Line service provide by Line)

$\mathrm{v}_{\mathrm{i}} \rightarrow$ relative perceived usefulness (perceived usefulness of Line perceived usefulness of Wechat)

$\mathrm{u}_{\mathrm{i}} \rightarrow$ relative trust (trust of LINE - trust of Wechat)

Each index will be transformed into standardized value using the following equation: $\quad x-\bar{x} / \sigma$

Ex $:$ Repurchase $\left(\mathrm{x}_{\mathrm{i}}\right) \rightarrow \Delta x_{i t}-\overline{\Delta x}_{i t} / \sigma, t=1,2, \ldots 291$
} 


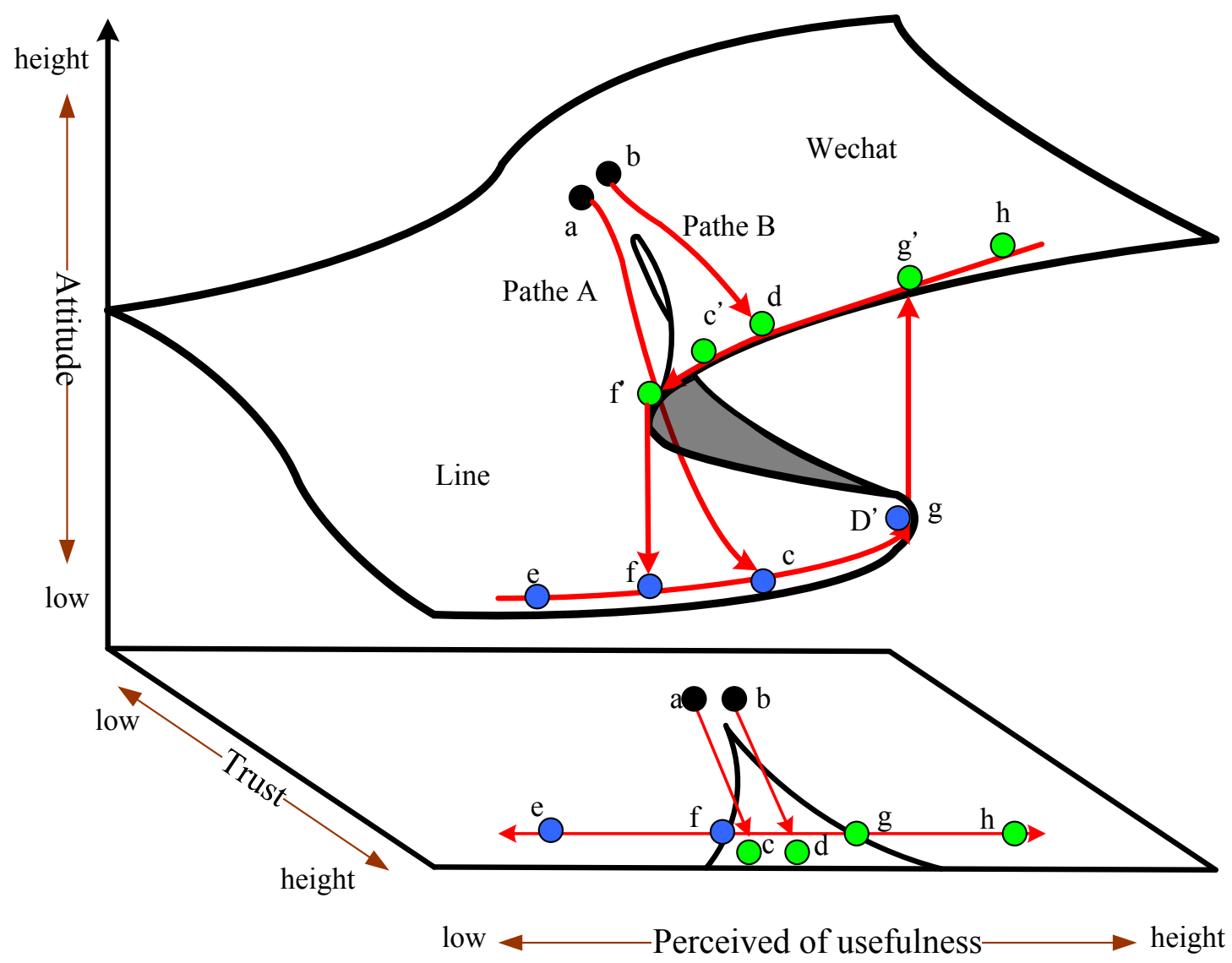

Figure 3. Plot of Trust versus Perceived Usefulness by K

\section{Discussions and Managerial Implications}

The Internet currently plays an important role as a business medium. Recently, there has been a dramatic proliferation in the number of Lines, based on the theory of reasoned action, we developed a model involving technology acceptance model. A large sample survey of online trading investors was employed to empirically examine this research model. A survey of 291 Line participants found strong support for the model. The data were analyzed using the two-step approach. First, confirmatory factor analysis (CFA) was performed to determine whether the measured variables reliably reflected the hypothesized latent variables. Second, structural models were tested to determine overall model fit and path coefficients. Finally, a cusp model of consumer behavior was developed to describe the effects of both trust and perceived usefulness on attitude of use of Line.

This empirical study demonstrates that higher perceived usefulness would lead customers to increase the attitude to switch to Line.com, even though the trust is higher. When the perceived usefulness is set at a lower level, drivers tend to continue choosing Wechat.com. This means that with the perceived usefulness strategy we are unable to reach the anticipated outcome. If the value of perceived usefulness is set at the pleat, the decision of customers in the behavioral system is ambiguous with a higher trust. Several significant catastrophe characteristics, such as divergence, catastrophe, hysteresis, and bimodality can be found in the behavioral models. In addition, online shopping operators should consider how to lower the customers' trust.

The results of this paper should assist Line providers to judge the effects of the perceived usefulness and realize customers' discontinuous attitude. We initially proposed the catastrophe model to describe customers' nonlinear model of customer attitude. Future research should be conducted to collect more relevant factors and to discuss their effects on behavioral decisions in order to more explicitly explain customer loyalty of Line provider.

\section{Acknowledgements}

This work was supported by the Nanhua University through a research grant (Y103000969).

\section{REFERENCES}

[1] Adams, D.A., Nelson, R.R., Todd, P.A. (1992) Perceived 
usefulness, ease of use, and usage of information technology: a replication, MIS Quarterly, 16, 227-247.

[2] Fornell, C., Larcker, D. (1981) Evaluating structural equation models with unobservable and measurement error, Journal of Marketing Research 18, 39-50.

[3] Hsu, C. L. and Lon, J. C. (2008) Acceptance of Line usage: The roles of technology acceptance, social influence and knowledge sharing motivation, Information \& Management, 45, 65-74.

[4] IP, R. K. and Wagner, C. (2008) Weblogging: A study of social computing and its impact on organizations, Decision
Support Systems, 242-250.

[5] Oliver, R. L., Rust, R., Varki, S. (1997) Customer delight: foundations, findings and managerial insight, Journal of Retailing, 73, 311-336.

[6] Oliva, T. A., Oliver R. L. and MacMillian, I. C. (1992) A Catastrophe Model for Developing Service Satisfaction Strategies, Journal of Marketing, 56, 83-95.

[7] Wang, Y., Qualls, W. (2007) Towards a theoretical model of technology adoption in hospitality organizations, International Journal of Hospitality Management, 26, 560573. 